\title{
Colds, flu and coughing: a review of over-the-counter cold and flu medicines
}

\author{
J Van Schoor \\ Amayeza Information Centre \\ *Corresponding author, email:jacky@amayeza-info.co.za
}

\begin{abstract}
The common cold is the most frequent human illness, and may be caused by several families of viruses, particularly the more than 100 serotypes of rhinoviruses. Inaccurate perceptions that antibiotics improve patient outcomes fuel the number of doctor visits and requests for antibiotics. The inappropriate use of antibiotics for minor, self-limiting, usually viral, upper-respiratory tract infections does not alter the course of the disease, and adds to the burden of antibiotic resistance. In addition, there is also no evidence to suggest that antibiotics prevent secondary bacterial complications following viral upper-respiratory tract infections. While most over-the-counter cold and flu remedies have no proven efficacy, they appear to attenuate the immune response to the infecting virus, and there is little doubt that appropriate symptomatic treatment can make the patient feel better. Therefore, symptomatic therapy remains the mainstay of common cold treatment. This article briefly reviews the components of cold and flu remedies, and provides a symptom-based assessment for the selection of appropriate over-the-counter medicine.
\end{abstract}

Keywords: colds, flu, coughing

Previously published: S Afr Fam Pract 2013;55(4):334-336. Updated by author.

\section{Introduction}

The constellation of a sore throat, rhinorrhoea, nasal congestion, coughing, low-grade fever, headaches and the malaise of the common cold, has been recognised as a disease entity since antiquity. ${ }^{1,2}$ The common cold is the most frequent human illness, and is caused by members of several families of viruses. The most likely offenders are the \pm 100 serotypes of rhinoviruses. ${ }^{2}$

Many individuals seek medical care for uncomplicated upperrespiratory tract infections. ${ }^{2} \mathrm{~A}$ study conducted in US children revealed that $30 \%$ of these visits result in a prescription for antibiotics. Inaccurate perceptions that colds are caused by bacteria, or that antibiotics improve the outcome, fuel the number of visits and parental requests for antibiotics. ${ }^{3}$ However, educational interventions by all healthcare professionals need to incorporate the suggestion that there is no cure for the common cold, other than allowing for the passage of time during which the infecting virus is cleared by the immune system. Indeed, it is the person's immune response, rather than the infecting virus, that is responsible for most of the symptoms of the common cold. ${ }^{1}$

Antiviral therapy is not available for most of the viruses that cause the common cold. ${ }^{2}$ The inappropriate use of antibiotics for minor, self-limiting, usually viral, upper-respiratory tract infections does not alter the course of the disease, but instead adds to the burden of antibiotic resistance. ${ }^{2,4}$ There is also no evidence to suggest that antibiotics prevent secondary bacterial complications of viral upper-respiratory tract infections. ${ }^{1}$
An appreciation that clear nasal secretions frequently become purulent without signifying bacterial infection, and that coughing is a normal symptom, is most important. Healthcare professionals should be familiar with the natural history of the common cold, so that any deviation from the norm is managed effectively. ${ }^{4}$ See Figure 1. The common cold is to be distinguished from influenza, pharyngitis, acute bronchitis, acute bacterial sinusitis, allergic rhinitis, and pertussis. ${ }^{5}$

\section{Treating cold and flu symptoms}

Most cold and flu medicines attenuate the effects of the person's immune response to the virus. ${ }^{1}$ While most over- the-counter (OTC) cold medicines have no proven efficacy and potential for serious adverse effects, ${ }^{2}$ there is little doubt that appropriate symptomatic treatment can make the patient feel better. ${ }^{5}$ The placebo effect also plays an important role here. ${ }^{5}$

Therefore, symptomatic therapy remains the mainstay of common cold treatment, although a number of agents have been investigated in an effort to shorten the duration of the illness. ${ }^{6}$ However, symptomatic therapies have associated risks, particularly in young children. ${ }^{2}$

The recommendation from the US Food and Drug Administration (FDA) is that OTC medications should not be used to treat the symptoms of the common cold in children who are younger than six years of age. 


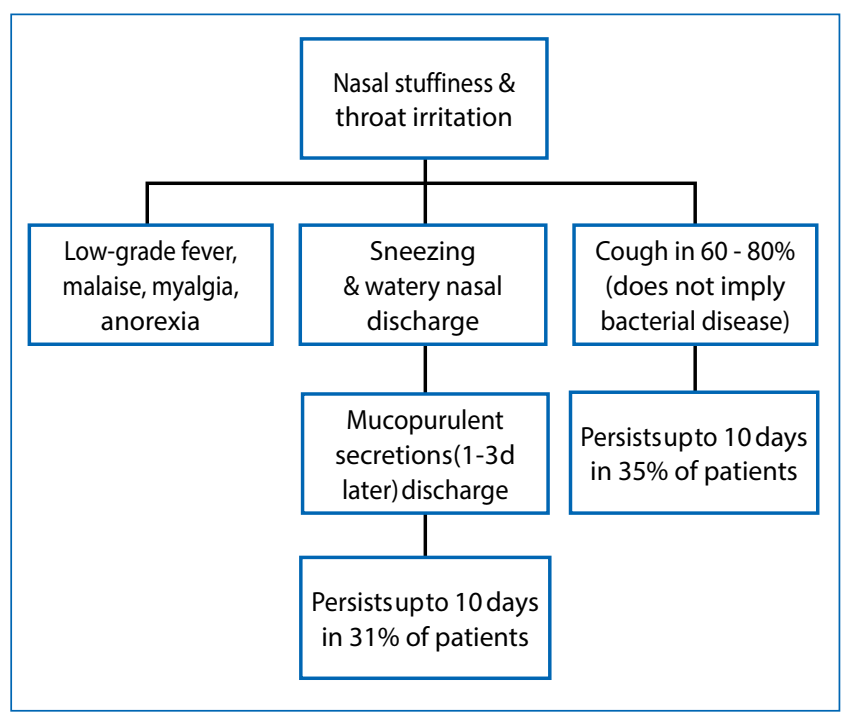

Adapted from S Afr Fam Pract. 2009;51(2):106

Figure 1: Natural history of the common cold
Symptoms need not be treated unless they bother the child, e.g. by interrupting sleep, interfering with eating and drinking, or by causing discomfort.

If an OTC medicine is required for a child, it is recommended that single-ingredient medicines are used to treat the most troublesome symptom, and that proper dosing instructions are followed to avoid potential toxicity. ${ }^{2}$

Polypharmacy abounds in the area of cold and flu remedies, and patients should not be overtreated. The recommended approach for adults is to select an appropriate treatment based on the patient's symptoms and available evidence, taking into account patient preferences $^{5}$ (See Table I).

\section{Symptom alleviators for colds and flu}

\section{Antihistamines}

Antihistamines may reduce some of the symptoms of a cold, such as rhinorrhoea and sneezing. These effects are due to the

Table I: Symptom-based assessment for over-the-counter cold and flu medicines

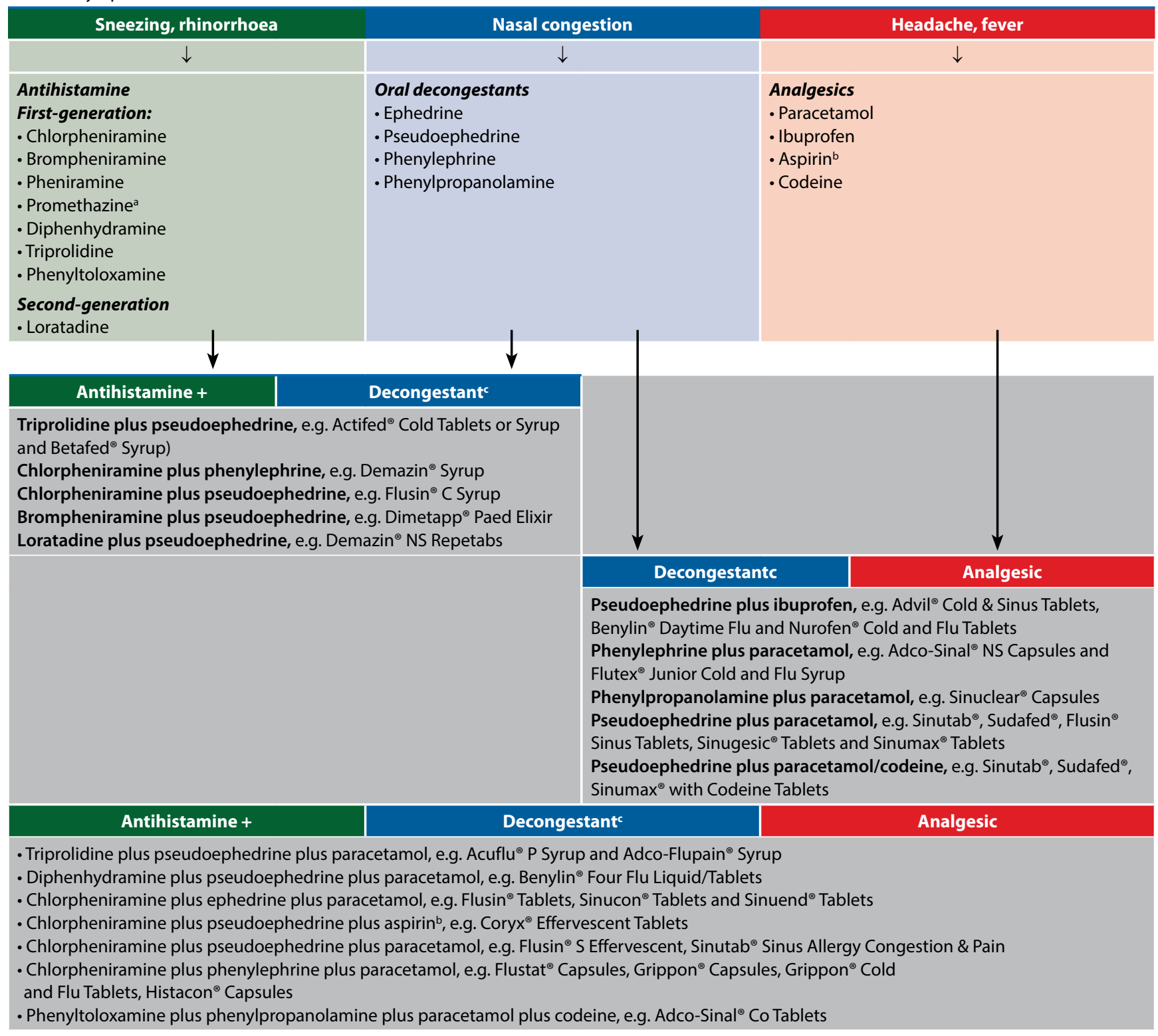

$a=$ Avoid giving to children under the age of 2 years, $b=$ Do not give aspirin to children under the age of 16 years, $c=$ Some products may also contain low doses of caffeine or vitamin $C$ 
anticholinergic effects of the older or first-generation antihistamines, such as chlorpheniramine, brompheniramine and promethazine. ${ }^{5}$ However, antihistamines are not effective in reducing nasal congestion. Some first-generation antihistamines, e.g. diphenhydramine, are included in cold remedies for their antitussive action, or to help the patient sleep, when included in combination products intended to be taken at night. ${ }^{5}$

Evidence suggests that when used alone, antihistamines are of little benefit in treating the symptoms of the common cold, but that they may offer limited symptomatic relief when used in combination with decongestants, analgesics and cough suppressants. ${ }^{5}$ Nonsedating antihistamines, such as loratadine, have been studied in combination with decongestants, and appear to decrease rhinorrhoea, sneezing and congestion, without causing drowsiness. ${ }^{1}$

Note: Promethazine-containing medicines are contraindicated for use in children under the age of two. ${ }^{7}$

\section{Decongestants}

Nasal congestion that is associated with the common cold may be alleviated by topical and oral sympathomimetic (adrenergic) decongestants, such as Xylometazoline, oxymetazoline, ephedrine, phenylephrine and pseudoephedrine. ${ }^{5}$ Compared to placebo, a single dose of an oral or topical decongestant improves subjective nasal symptoms that are associated with a reduction in airways resistance. ${ }^{6}$ However, topical decongestant use should be limited to two to three days, as rebound congestion may occur after 72 hours of use. ${ }^{5,6}$

Oral sympathomimetic decongestants, such as pseudoephedrine, are no longer available OTC in South Africa as single-ingredient products.

Various combination products are available (See Table I) ${ }^{8}$ :

- An oral decongestant in combination with a first- or secondgeneration antihistamine.

- An oral decongestant in combination with an analgesic, such as paracetamol, or a nonsteroidal anti- inflammatory drug, such as ibuprofen.

- An oral decongestant in combination with both an antihistamine and an analgesic.

\section{Other components of cold and flu remedies}

For many years, vitamin $\mathrm{C}$ has been touted as a therapeutic agent for the prevention and treatment of the common cold. ${ }^{1}$ Highdose vitamin $C$ ( $>1 \mathrm{~g} /$ day) has been shown to reduce the duration of colds by eight per cent. ${ }^{5}$ However, the improvement in symptoms following the intake of high- dose vitamin $\mathrm{C}$ appears to be no greater than that seen with standard OTC cold remedies. ${ }^{1}$

Echinacea is a popular herbal cold remedy. Few modern studies support its efficacy. Prophylaxis studies suggest that echinacea has no significant effect in preventing rhinovirus infection. ${ }^{1}$ Treatment studies show mixed results, with some studies showing some improvement in symptoms, while others show no improvement compared to placebo. ${ }^{1}$

Caffeine is included in some combination products to produce wakefulness and to offset some of the sedation caused by the firstgeneration antihistamine. ${ }^{5}$ Doses of at least $100 \mathrm{mg}$ are needed to produce such an effect. Most OTC products contain 30-50 mg per tablet, about the same as a cup of coffee. ${ }^{5}$ It has also been claimed that caffeine increases the effectiveness of analgesics, but the evidence to support this claim is not definitive. ${ }^{5}$

\section{References}

1. Hay CM. Treatment and prevention of rhinovirus infections. UpToDate [homepage on the Internet]. Available from: www.uptodate.com

2. Pappas $\mathrm{DE}$, Hendley JO. The common cold in children: treatment and prevention UpToDate [homepage on the Internet]. Available from: http://www.uptodate. $\mathrm{com} /$ contents/the-common-cold-in-children-treatment-and-prevention

3. Lee GM, Friedman JF, Ross-Degnan D, et al. Misconceptions about colds and predictors of health service utilization. Pediatrics. 2003;111(2):231-236.

4. Working Group of the Infectious Diseases Society of Southern Africa. Updated guideline for the management of upper respiratory tract infections in South Africa: 2008. S Afr J Epidemiol Infect. 2008;23(4):27-40.

5. Blenkinsopp A, Paxton P, Blenkinsopp J. Symptoms in the pharmacy. A guide to the management of common illness. 6th ed. West Sussex: Wiley-Blackwell; 2009.

6. Sexton DJ. The common cold in adults: treatment and prevention. UpToDate [homepage on the Internet]. 2018. Available from: http://www. uptodate.com/ contents/the-common-cold-in-adults-treatment-and-prevention

7. Medicine safety alert: promethazine contraindicated in children under 2 years of age. Medicines Control Council. Pretoria: MSA Promethazine; 2007 [homepage on the Internet]. Available from: www.mccza. com/.../21.2_MSA_Promethazine_Apr07_v1.doc

8. MIMS. 2018;58(3) 